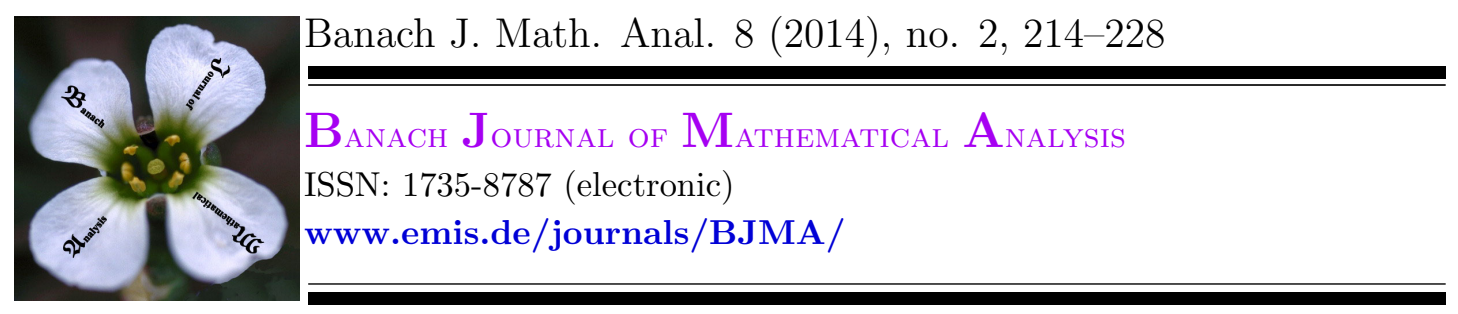

\title{
SEMI-NORMAL STRUCTURE AND BEST PROXIMITY PAIR RESULTS IN CONVEX METRIC SPACES
}

\author{
MOOSA GABELEH \\ Dedicated to my late friend Hassan Shams (1981-2004), an outstanding math student \\ Communicated by R. E. Curto.

\begin{abstract}
A new geometric notion on a nonempty and convex pair of subsets of a convex metric space $X$, called semi-normal structure, is introduced and used to investigate the existence of best proximity pairs for a new class of mappings, called strongly noncyclic relatively C-nonexpansive. We also study the structure of minimal sets of strongly noncyclic relatively C-nonexpansive mappings in the setting of convex metric spaces.
\end{abstract}

\section{INTRODUCTION}

Let $(X, d)$ be a metric space. A self-mapping $T: X \rightarrow X$ is called $C$-contraction if there exists $\alpha \in\left[0, \frac{1}{2}\right)$ such that

$$
d(T x, T y) \leq \alpha[d(x, T y)+d(y, T x)],
$$

for all $x, y \in X$. The class of C-contractions was introduced by Chatterjea in [8].

It was proved in [8] that if $X$ is complete metric space, every C-contraction selfmapping defined on $X$ has a unique fixed point ([8]). Note that the C-contraction self-mappings may not be continuous.

We say that a self-mapping $T: X \rightarrow X$ is $C$-nonexpansive if

$$
d(T x, T y) \leq \frac{1}{2}[d(x, T y)+d(y, T x)] \quad \forall x, y \in X .
$$

Date: Received: Sep. 13, 2013; Accepted: Dec. 29, 2013.

2010 Mathematics Subject Classification. Primary 47H10; Secondary 47H09, 46B20.

Key words and phrases. Best proximity pair, semi-normal structure, strongly noncyclic relatively C-nonexpansive, convex metric space. 
Now, let $A$ and $B$ be two nonempty subsets of a metric space $(X, d)$. A mapping $T: A \cup B \rightarrow A \cup B$ is said to be a noncyclic mapping provided that $T(A) \subseteq A$ and $T(B) \subseteq B$. For this class of mappings, we consider the following minimization problem: Find

$$
\min _{x \in A} d(x, T x), \quad \min _{y \in B} d(y, T y) \quad \text { and } \min _{(x, y) \in A \times B} d(x, y) .
$$

A point $(p, q) \in A \times B$ is said to be a best proximity pair of the noncyclic mapping $T$ provided that $(p, q)$ is a solution of the above minimization problem, that is,

$$
T p=p, \quad T q=q \quad \text { and } \quad d(p, q)=\operatorname{dist}(A, B),
$$

where, $\operatorname{dist}(A, B):=\inf \{d(x, y):(x, y) \in A \times B\}$. We mention that in [1], the authors investigated sufficient conditions to ensure the existence of best proximity pairs for noncyclic mappings.

Let $(A, B)$ be a nonempty pair of subsets of a metric space $(X, d)$. A mapping $T: A \cup B \rightarrow A \cup B$ is said to be noncyclic relatively nonexpansive if $T$ is noncyclic and $d(T x, T y) \leq d(x, y)$ for all $(x, y) \in A \times B$.

Eldred, Kirk and Veeramani ([5]) established the existence of a best proximity pair for noncyclic relatively nonexpansive mappings by using a geometric notion of proximal normal structure in the setting of Banach spaces.

We shall say that a pair $(A, B)$ in a metric space $(X, d)$ satisfies a property if both $A$ and $B$ satisfy that property. For instance, $(A, B)$ is closed if and only if both $A$ and $B$ are closed; $(A, B) \subseteq(C, D) \Leftrightarrow A \subseteq C$, and $B \subseteq D$. We shall also adopt the following notations.

$$
\begin{gathered}
\delta_{x}(A):=\sup \{d(x, y): y \in A\} \text { for all } x \in X, \\
\delta(A, B):=\sup \{d(x, y): x \in A, y \in B\}, \\
\operatorname{diam}(A):=\delta(A, A) . \\
A_{0}:=\{x \in A: d(x, y)=\operatorname{dist}(A, B) \text { for some } y \in B\}, \\
B_{0}:=\{y \in B: d(x, y)=\operatorname{dist}(A, B) \text { for some } x \in A\} .
\end{gathered}
$$

We note that if $A$ and $B$ are nonempty, weakly compact and convex subsets of a Banach space $X$, then $\left(A_{0}, B_{0}\right)$ must be a nonempty pair in $X$.

Definition 1.1. A Banach space $X$ is said to be

(i) uniformly convex if there exists a strictly increasing function $\delta:(0,2] \rightarrow[0,1]$ such that the following implication holds for all $x, y, p \in X, R>0$ and $r \in[0,2 R]$ :

$$
\left\{\begin{array}{l}
\|x-p\| \leq R, \\
\|y-p\| \leq R, \quad \Rightarrow\left\|\frac{x+y}{2}-p\right\| \leq\left(1-\delta\left(\frac{r}{R}\right)\right) R ; \\
\|x-y\| \geq r
\end{array}\right.
$$

(ii) strictly convex if the following implication holds for all $x, y, p \in X$ and $R>0$ :

$$
\left\{\begin{array}{l}
\|x-p\| \leq R \\
\|y-p\| \leq R, \\
x \neq y
\end{array} \quad \Rightarrow\left\|\frac{x+y}{2}-p\right\|<R\right.
$$


In the current paper, we introduce a new class of mappings, called strongly noncyclic relatively $C$-nonexpansive and study sufficient conditions which ensure the existence of best proximity pairs for this class of mappings in the setting of convex metric spaces. Moreover, we survey the structure of minimal sets for this class of noncyclic mappings and show that results alike to the celebrated GoebelKarlovitz Lemma $([6,10,11])$ for nonexpansive self-mappings can be obtained for strongly noncyclic relatively C-nonexpansive mappings.

\section{Preliminaries}

In [17], Takahashi introduced the notion of convexity in metric spaces as follows.

Definition 2.1. Let $(X, d)$ be a metric space and $I:=[0,1]$. A mapping $\mathcal{W}$ : $X \times X \times I \rightarrow X$ is said to be a convex structure on $X$ provided that for each $(x, y ; \lambda) \in X \times X \times I$ and $u \in X$,

$$
d(u, \mathcal{W}(x, y ; \lambda)) \leq \lambda d(u, x)+(1-\lambda) d(u, y) .
$$

A metric space $(X, d)$ together with a convex structure $\mathcal{W}$ is called a convex metric space, which is denoted by $(X, d, \mathcal{W})$. A Banach space and each of its convex subsets are convex metric spaces. But a Frechet space is not necessary a convex metric space. The other examples of convex metric spaces which are not imbedded in any Banach space can be founded in [17].

To describe our results, we need some definitions and preliminary facts from the reference [17].

Definition 2.2. A subset $K$ of a convex metric space $(X, d, \mathcal{W})$ is said to be a convex set provided that $\mathcal{W}(x, y ; \lambda) \in K$ for all $x, y \in K$ and $\lambda \in I$.

Proposition 2.3. Let $(X, d, \mathcal{W})$ be a convex metric space and let $\mathcal{B}(x ; r)$ denote the closed ball centered at $x \in X$ with radius $r \geq 0$. Then $\mathcal{B}(x ; r)$ is a convex subset of $X$.

Proposition 2.4. Let $\left\{K_{\alpha}\right\}_{\alpha \in A}$ be a family of convex subsets of $X$, then $\bigcap_{\alpha \in A} K_{\alpha}$ is also a convex subset of $X$.

Definition 2.5. A convex metric space $(X, d, \mathcal{W})$ is said to have property $(C)$ if every bounded decreasing net of nonempty, closed and convex subsets of $X$ has a nonempty intersection.

For example every weakly compact convex subset of a Banach space has property $(\mathrm{C})$. The next example ensures that condition $(\mathrm{C})$ is natural as well in the metrical setting.

Example 2.6. ([15]) Let $\mathcal{H}$ be a Hilbert space and let $X$ be a nonempty closed subset of $\{x \in \mathcal{H}:\|x\|=1\}$ such that if $x, y \in X$ and $\alpha, \beta \in[0,1]$ with $\alpha+\beta=1$, then $\frac{\alpha x+\beta y}{\|\alpha x+\beta y\|} \in X$ and $\operatorname{diam}(X) \leq \frac{\sqrt{2}}{2}$, where $\operatorname{diam}(X):=\sup \{d(x, y): x, y \in$ $X\}$. Let $d(x, y):=\cos ^{-1}(\langle x, y\rangle)$ for all $x, y \in X$, where $\langle$,$\rangle is the inner$ product of $\mathcal{H}$. If we define the convex structure $\mathcal{W}: X \times X \times I \rightarrow X$ with $\mathcal{W}(x, y, \lambda):=\frac{\lambda x+(1-\lambda) y}{\|\lambda x+(1-\lambda) y\|}$, then $(X, d)$ is a complete convex metric space which has the property (C) (for more information see Example 2 of [15]). 
Definition 2.7. ([9]) A convex metric space $(X, d, \mathcal{W})$ is said to have property (D) provided that for each $x_{1}, x_{2}, y_{1}, y_{2}$ in $X$ we have

$$
d\left(\mathcal{W}\left(x_{1}, x_{2}, \frac{1}{2}\right), \mathcal{W}\left(y_{1}, y_{2}, \frac{1}{2}\right)\right)<\frac{1}{2}\left[d\left(x_{1}, y_{1}\right)+d\left(x_{2}, y_{2}\right)\right]
$$

It is clear that every strictly convex Banach space is a convex metric space which satisfies the property (D).

Let $A$ be a nonempty subset of a convex metric space $(X, d, \mathcal{W})$. The closed and convex hull of a set $A$ will be denoted by $\overline{\operatorname{cov}}(A)$ and defined as below.

$\overline{\operatorname{cov}}(A):=\bigcap\{C: C$ is a closed and convex subset of $X$ such that $C \supseteq A\}$.

The next lemmas will be used in our results.

Lemma 2.8. ([2]) Let $\left(K_{1}, K_{2}\right)$ be a nonempty pair of a convex metric space $(X, d, \mathcal{W})$. Then $\delta\left(K_{1}, K_{2}\right)=\delta\left(\overline{\operatorname{cov}}\left(K_{1}\right), \overline{\operatorname{cov}}\left(K_{2}\right)\right)$.

Lemma 2.9. ([12]) Let $A$ be a nonempty subset of a convex metric space $(X, d, \mathcal{W})$. Then

$$
\delta_{x}(A)=\delta_{x}(\overline{\operatorname{cov}}(A)), \quad \forall x \in X
$$

Definition 2.10. Let $A$ be a nonempty subset in a metric space $(X, d)$. A point $p$ in $A$ is said to be a diametral point if $\delta_{p}(A)=\operatorname{diam}(A)$.

The notion of normal structure was introduced by Brodskil and Milman in [7] and then it was generalized by Takahashi in convex metric spaces as follows.

Definition 2.11. ([17]) A convex metric space $(X, d, \mathcal{W})$ is said to have normal structure if for each bounded, closed and convex subset $K$ of $X$ which contains at least two points, there exists an element $p \in K$ which is a nondiametral point.

By using this geometric notion the following fixed point theorem was established in [17].

Theorem 2.12. Suppose that $(X, d, \mathcal{W})$ is a convex metric space such that $X$ has the property $(C)$. Let $K$ be a nonempty bounded, closed and convex subset of $X$ with normal structure. If $T: K \rightarrow K$ is a nonexpansive mapping, that is,

$$
d(T x, T y) \leq d(x, y), \quad \forall x, y \in K
$$

then $T$ has a fixed point in $K$.

We mention that the previous theorem is an extension of Kirk's fixed point theorem ([13]) in the setting of convex metric spaces.

Some of interesting results regarding the existence and convergence of fixed points for various classes of nonexpansive mappings can be found in [3, 4, 14, 16$]$.

Definition 2.13. ([9]) Let $(A, B)$ be a nonempty pair of subsets of a metric space $(X, d)$. We say that the pair $(A, B)$ is a proximal compactness pair provided that every net $\left(\left\{x_{\alpha}\right\},\left\{y_{\alpha}\right\}\right)$ of $A \times B$ satisfying the condition that $d\left(x_{\alpha}, y_{\alpha}\right) \rightarrow$ $\operatorname{dist}(A, B)$, has a convergent subnet in $A \times B$.

It is clear that if $(A, B)$ is a compact pair in a metric space $(X, d)$ then $(A, B)$ is proximal compactness. 


\section{Best PROXIMity PAIR RESUlts}

We begin our main result of this section with the following geometric notion.

Definition 3.1. A convex pair $\left(K_{1}, K_{2}\right)$ in a convex metric space $(X, d, \mathcal{W})$ is said to have semi-normal structure if for any bounded, closed and convex pair $\left(H_{1}, H_{2}\right) \subseteq\left(K_{1}, K_{2}\right)$ for which $\delta\left(H_{1}, H_{2}\right)>\operatorname{dist}\left(H_{1}, H_{2}\right)$ and $\operatorname{dist}\left(H_{1}, H_{2}\right)=$ $\operatorname{dist}\left(K_{1}, K_{2}\right)$, there exits $\left(x_{1}, x_{2}\right) \in H_{1} \times H_{2}$ such that

$$
d\left(x_{1}, x_{2}\right)=\operatorname{dist}\left(K_{1}, K_{2}\right) \quad \& \quad \max \left\{\delta_{x_{1}}\left(H_{2}\right), \delta_{x_{2}}\left(H_{1}\right)\right\}<\delta\left(H_{1}, H_{2}\right) .
$$

Note that if in above definition $K_{1}=K_{2}$, then $\left(K_{1}, K_{2}\right)$ has semi-normal structure if and only if the set $K_{1}$ has normal structure in the sense of Brodskil and Milman ([7]).

Definition 3.2. Let $(A, B)$ be a nonempty pair of subsets of a convex metric space $(X, d, \mathcal{W})$. A mapping $T: A \cup B \rightarrow A \cup B$ is said to be strongly noncyclic relatively $\mathrm{C}$-nonexpansive provided that $T$ is noncyclic and

$$
d(T x, T y)=d(x, y), \forall(x, y) \in A \times B \quad \text { with } \quad d(x, y)=\operatorname{dist}(A, B),
$$

and

$d(T x, T y) \leq \min \{d(x, T y), d(y, T x)\}, \forall(x, y) \in A \times B$ with $d(x, y)>\operatorname{dist}(A, B)$.

Definition 3.3. Let $(A, B)$ be a nonempty pair of subsets of a convex metric space $(X, d, \mathcal{W})$. A mapping $T: A \cup B \rightarrow A \cup B$ is said to be noncyclic relatively C-nonexpansive provided that $T$ is noncyclic and

$$
d(T x, T y)=d(x, y), \forall(x, y) \in A \times B \quad \text { with } \quad d(x, y)=\operatorname{dist}(A, B),
$$

and

$$
d(T x, T y) \leq \frac{1}{2}[d(x, T y)+d(y, T x)], \forall(x, y) \in A \times B \text { with } d(x, y)>\operatorname{dist}(A, B)
$$

It is clear that the class of noncyclic relatively C-nonexpansive mappings contains the class of strongly noncyclic relatively C-nonexpansive mappings as a subclass.

The next theorem guarantees the existence of best proximity pairs for strongly noncyclic relatively C-nonexpansive mappings in convex metric spaces.

Theorem 3.4. Let $(A, B)$ be a nonempty, bounded, closed and convex pair in a convex metric space $(X, d, \mathcal{W})$ such that $X$ has the properties $(C)$ and $(D), A_{0}$ is nonempty and $(A, B)$ is a proximal compactness pair. Suppose that $T: A \cup B \rightarrow$ $A \cup B$ is a strongly noncyclic relatively $C$-nonexpansive mapping. If $(A, B)$ has semi-normal structure, then $T$ has a best proximity pair.

Proof. Suppose that $\mathfrak{F}$ denote the collection of all nonempty, closed and convex pairs $(E, F)$ which are subsets of $(A, B)$ and such that $T$ is noncyclic on $E \cup F$ and $d(x, y)=\operatorname{dist}(A, B)$ for some $(x, y) \in E \times F$. Since $A_{0}$ is nonempty, $(A, B) \in \mathfrak{F}$. Also, $\mathfrak{F}$ is partially ordered by revers inclusion, that is, $\left(E_{1}, F_{1}\right) \preceq\left(E_{2}, F_{2}\right) \Leftrightarrow$ $\left(E_{2}, F_{2}\right) \subseteq\left(E_{1}, F_{1}\right)$. Let $\left\{\left(E_{\alpha}, F_{\alpha}\right)\right\}_{\alpha}$ be a descending chain in $\mathfrak{F}$. Put $E:=\bigcap E_{\alpha}$ and $F:=\bigcap F_{\alpha}$. By the fact that $X$ has the property $(\mathrm{C})$, we deduce that $(E, F)$ is a nonempty pair and we know that $(E, F)$ is closed. It is easy to see that $T$ 
is noncyclic on $E \cup F$. Moreover, by Proposition 2.4, $(E, F)$ is a convex pair. Now, let $\left(x_{\alpha}, y_{\alpha}\right) \in E_{\alpha} \times F_{\alpha}$ be such that $d\left(x_{\alpha}, y_{\alpha}\right)=\operatorname{dist}(A, B)$. Since $(A, B)$ is proximal compactness, $\left(x_{\alpha}, y_{\alpha}\right)$ has a convergent subsequence say $\left(x_{\alpha_{i}}, y_{\alpha_{i}}\right)$ such that $x_{\alpha_{i}} \rightarrow x \in A$ and $y_{\alpha_{i}} \rightarrow y \in B$. Hence,

$$
d(x, y)=\lim _{i} d\left(x_{\alpha_{i}}, y_{\alpha_{i}}\right)=\operatorname{dist}(A, B) .
$$

Therefore, there exists an element $(x, y) \in E \times F$ such that $d(x, y)=\operatorname{dist}(A, B)$. So, every increasing chain in $\mathfrak{F}$ is bounded above with respect to revers inclusion relation. Then by using Zorn's Lemma we obtain a minimal element for $\mathfrak{F}$, say $\left(K_{1}, K_{2}\right)$. Suppose that

$$
\delta\left(K_{1}, K_{2}\right)=\operatorname{dist}\left(K_{1}, K_{2}\right)(=\operatorname{dist}(A, B)) .
$$

Then for each $(x, y) \in\left(K_{1}, K_{2}\right)$ we have $d(x, y)=\operatorname{dist}\left(K_{1}, K_{2}\right)$. We now claim that both $K_{1}$ and $K_{2}$ are singleton. Assume that $x_{1}, x_{2} \in K_{1}$ such that $x_{1} \neq x_{2}$. Then for all $y_{1}, y_{2} \in K_{2}$ we have

$$
d\left(x_{1}, y_{1}\right)=d\left(x_{2}, y_{2}\right)=\operatorname{dist}(A, B) .
$$

Since $\left(K_{1}, K_{2}\right)$ is a convex pair, $\mathcal{W}\left(x_{1}, x_{2}, \frac{1}{2}\right) \in K_{1}$ and $\mathcal{W}\left(y_{1}, y_{2}, \frac{1}{2}\right) \in K_{2}$. By the fact that $X$ has the property (D) we conclude that

$$
\begin{gathered}
\operatorname{dist}(A, B) \leq d\left(\mathcal{W}\left(x_{1}, x_{2}, \frac{1}{2}\right), \mathcal{W}\left(y_{1}, y_{2}, \frac{1}{2}\right)\right) \\
<\frac{1}{2}\left[d\left(x_{1}, y_{1}\right)+d\left(x_{2}, y_{2}\right)\right]=\operatorname{dist}(A, B),
\end{gathered}
$$

which is a contradiction. Hence, $K_{1}$ is singleton. Similarly, we can see that $K_{2}$ is a singleton set. This implies that the noncyclic mapping $T$ has a best proximity pair in this case and we are finished. Now, suppose that

$$
\delta\left(K_{1}, K_{2}\right)>\operatorname{dist}\left(K_{1}, K_{2}\right)(=\operatorname{dist}(A, B)) .
$$

Let $(p, q) \in K_{1} \times K_{2}$ be such that $d(p, q)=\operatorname{dist}(A, B)$. Consider the nonempty, closed and convex pair $\left(\overline{\operatorname{cov}}\left(T\left(K_{1}\right)\right), \overline{\operatorname{cov}}\left(T\left(K_{2}\right)\right)\right) \subseteq\left(K_{1}, K_{2}\right)$. We have $(T p, T q) \in$ $\left(\overline{\operatorname{cov}}\left(T\left(K_{1}\right)\right), \overline{\operatorname{cov}}\left(T\left(K_{2}\right)\right)\right)$, and so,

$$
\operatorname{dist}(A, B) \leq \operatorname{dist}\left(\overline{\operatorname{cov}}\left(T\left(K_{1}\right)\right), \overline{\operatorname{cov}}\left(T\left(K_{2}\right)\right)\right) \leq d(T p, T q)=d(p, q)=\operatorname{dist}(A, B) .
$$

Also,

$$
T\left(\overline{\operatorname{cov}}\left(T\left(K_{1}\right)\right)\right) \subseteq T\left(K_{1}\right) \subseteq \overline{\operatorname{cov}}\left(T\left(K_{1}\right)\right),
$$

and similarly, $T\left(\overline{\operatorname{cov}}\left(T\left(K_{2}\right)\right)\right) \subseteq \overline{\operatorname{cov}}\left(T\left(K_{2}\right)\right)$. Therefore, the mapping

$$
T: \overline{\operatorname{cov}}\left(T\left(K_{1}\right)\right) \cup \overline{\operatorname{cov}}\left(T\left(K_{2}\right)\right) \rightarrow \overline{\operatorname{cov}}\left(T\left(K_{1}\right)\right) \cup \overline{\operatorname{cov}}\left(T\left(K_{2}\right)\right)
$$

is noncyclic. So, $\left(\overline{\operatorname{cov}}\left(T\left(K_{1}\right)\right), \overline{\operatorname{cov}}\left(T\left(K_{2}\right)\right)\right) \in \mathfrak{F}$. It now follows from the fact that $\left(K_{1}, K_{2}\right)$ is the minimal element of $\mathfrak{F}$,

$$
K_{1}=\overline{\operatorname{cov}}\left(T\left(K_{1}\right)\right) \quad \& \quad K_{2}=\overline{\operatorname{cov}}\left(T\left(K_{2}\right)\right) .
$$

Put

$$
r_{1}:=\delta_{p}\left(K_{2}\right), \quad r_{2}:=\delta_{q}\left(K_{1}\right) \quad \text { and } \quad r:=\max \left\{r_{1}, r_{2}\right\} .
$$

Since $(A, B)$ has the semi-normal structure, we have

$$
r<\delta\left(K_{1}, K_{2}\right)
$$


Suppose that

$$
C_{r}\left(K_{2}\right):=K_{1} \bigcap\left(\cap_{x \in K_{2}} \mathcal{B}(x ; r)\right) \quad \& \quad C_{r}\left(K_{1}\right):=K_{2} \bigcap\left(\cap_{x \in K_{1}} \mathcal{B}(x ; r)\right) .
$$

Propositions 2.3 and 2.4 conclude that $\left(C_{r}\left(K_{2}\right), C_{r}\left(K_{1}\right)\right) \subseteq\left(K_{1}, K_{2}\right)$ is a nonempty, closed and convex pair. Besides, it is easy to see that for $(x, y) \in K_{1} \times K_{2}$,

$$
(x, y) \in C_{r}\left(K_{2}\right) \times C_{r}\left(K_{1}\right) \Leftrightarrow K_{2} \subseteq \mathcal{B}(x ; r), \quad K_{1} \subseteq \mathcal{B}(y ; r) .
$$

Also, $(p, q) \in C_{r}\left(K_{2}\right) \times C_{r}\left(K_{1}\right)$ which deduces that

$$
\operatorname{dist}\left(C_{r}\left(K_{2}\right), C_{r}\left(K_{1}\right)\right) \leq d(p, q)=\operatorname{dist}\left(K_{1}, K_{2}\right) \leq \operatorname{dist}\left(C_{r}\left(K_{2}\right), C_{r}\left(K_{1}\right)\right) .
$$

We now assert that $T$ is noncyclic on $C_{r}\left(K_{2}\right) \cup C_{r}\left(K_{1}\right)$. Let $u \in C_{r}\left(K_{2}\right)$. Since $T$ is strongly noncyclic relatively $\mathrm{C}$-nonexpansive, for each $v \in K_{2}$ we have

$$
d(T u, T v) \leq \min \{d(u, T v), d(v, T u)\} \leq r,
$$

which concludes that $T v \in B(T u ; r)$. Therefore, $T\left(K_{2}\right) \subseteq \mathcal{B}(T u ; r)$ and so,

$$
K_{2}=\overline{\operatorname{cov}}\left(T\left(K_{2}\right)\right) \subseteq \mathcal{B}(T u ; r),
$$

that is, $T u \in C_{r}\left(K_{2}\right)$. Thus, $T\left(C_{r}\left(K_{2}\right)\right) \subseteq C_{r}\left(K_{2}\right)$. Similarly, we can see that $T\left(C_{r}\left(K_{1}\right)\right) \subseteq C_{r}\left(K_{1}\right)$. Hence, $T$ is noncyclic on $C_{r}\left(K_{2}\right) \cup C_{r}\left(K_{1}\right)$. The minimality of $\left(K_{1}, K_{2}\right)$ implies that

$$
C_{r}\left(K_{2}\right)=K_{1} \quad \& \quad C_{r}\left(K_{1}\right)=K_{2} .
$$

So, $K_{1} \subseteq \bigcap_{v \in K_{2}} \mathcal{B}(v ; r)$. Then for each $u \in K_{1}$ we have $\delta_{u}\left(K_{2}\right) \leq r$. Thus,

$$
\delta\left(K_{1}, K_{2}\right)=\sup _{u \in K_{1}} \delta_{u}\left(K_{2}\right) \leq r
$$

which is a contradiction since $r<\delta\left(K_{1}, K_{2}\right)$.

The next corollary obtains from Theorem 3.4, immediately.

Corollary 3.5. Let $(A, B)$ be a nonempty, weakly compact and convex pair in a strictly convex Banach space $X$ such that $(A, B)$ has semi-normal structure. If $T: A \cup B \rightarrow A \cup B$ is a strongly noncyclic relatively $C$-nonexpansive mapping, then $T$ has a best proximity pair.

The next theorem guarantees the existence of best proximity pairs in uniformly convex Banach spaces.

Theorem 3.6. Let $(A, B)$ be a nonempty, bounded, closed and convex pair in a uniformly convex Banach space $X$. Suppose that $T: A \cup B \rightarrow A \cup B$ is a strongly noncyclic relatively $C$-nonexpansive mapping. Then $T$ has a best proximity pair.

Proof. Suppose that $T$ has not a best proximity pair. We get a contradiction by showing that $(A, B)$ has semi-normal structure. Let $\left(K_{1}, K_{2}\right)$ be a closed and convex subset of $(A, B)$ such that $\delta\left(K_{1}, K_{2}\right)>\operatorname{dist}\left(K_{1}, K_{2}\right)$ and $\operatorname{dist}\left(K_{1}, K_{2}\right)=$ $\operatorname{dist}(A, B)$. Let $(p, q) \in K_{1} \times K_{2}$ be such that $\|p-q\|=\operatorname{dist}\left(K_{1}, K_{2}\right)(=$ $\operatorname{dist}(A, B))$. Since $T$ is strongly noncyclic relatively $\mathrm{C}$-nonexpansive, we have

$$
\|T p-T q\|=\|p-q\|=\operatorname{dist}(A, B) .
$$


Now, we must have $p \neq T p$ or $q \neq T q$ by the fact that $T$ has not best proximity pair. It now follows from the strictly convexity of $X$ that

$$
\left\|\frac{p+T p}{2}-\frac{q+T q}{2}\right\|=\operatorname{dist}(A, B) .
$$

Put $R:=\delta\left(K_{1}, K_{2}\right)$ and $r:=\min \{\|p-T p\|,\|q-T q\|\}$. Also, set $p^{\star}:=\frac{p+T p}{2}$ and $q^{\star}:=\frac{T q+q}{2}$. Now, for all $y \in K_{2}$ we have

$$
\left\{\begin{array}{l}
\|p-y\| \leq R \\
\|T p-y\| \leq R, \\
\|p-T p\| \geq r .
\end{array}\right.
$$

Since $X$ is a uniformly convex Banach space, we conclude that

$$
\left\|p^{\star}-y\right\| \leq\left(1-\delta\left(\frac{r}{R}\right)\right) R, \quad \forall y \in K_{2},
$$

Hence, $\delta_{p^{\star}}\left(K_{2}\right)<R$. Similarly, we can see that $\delta_{q^{\star}}\left(K_{1}\right)<R$. Therefore,

$$
\left\|p^{\star}-q^{\star}\right\|=\operatorname{dist}(A, B) \quad \& \quad \max \left\{\delta_{p^{\star}}\left(K_{2}\right), \delta_{q^{\star}}\left(K_{1}\right)\right\}<\delta\left(K_{1}, K_{2}\right) .
$$

That is, $(A, B)$ has semi-normal structure.

Here, we prove a new fixed point theorem by using the geometric notion of normal structure in convex metric spaces.

Theorem 3.7. Suppose that $(X, d, \mathcal{W})$ is a convex metric space such that $X$ has the property $(C)$. Let $A$ be a nonempty, bounded, closed and convex subset of $X$ with normal structure. If $T: A \rightarrow A$ is a strongly $C$-nonexpansive, that is,

$$
d(T x, T y) \leq \min \{d(x, T y), d(y, T x)\} \quad \forall x, y \in A,
$$

then $T$ has a fixed point in $A$.

Proof. Invoking property (C) and Zorn's Lemma, we obtain a subset $K$ of $A$ which is minimal with respect to being nonempty, closed, convex and $T$-invariant. So, we must have $\overline{\operatorname{cov}}(T(K))=K$. Suppose that $\operatorname{diam}(K)>0$. By the fact that $X$ has normal structure there exist $p \in K$ and $r>0$ such that $\delta_{p}(K)<r<\operatorname{diam}(K)$. Put

$$
C:=\{x \in K: K \subseteq \mathcal{B}(x ; r)\} .
$$

Note that $p \in C$ and then $C$ is nonempty. Moreover, it is easy to see that

$$
C=K \bigcap\left(\cap_{x \in K} \mathcal{B}(x ; r)\right),
$$

that is, $C$ is a closed and convex subset of a convex metric space $X$. Now, let $x \in C$. Then for each $y \in K$ we have

$$
d(T x, T y) \leq \min \{d(x, T y), d(T x, y)\} \leq r .
$$

This implies that $T y \in \mathcal{B}(T x ; r)$ for each $y \in K$ and so,

$$
K=\overline{\operatorname{cov}}(T(K)) \subseteq \mathcal{B}(T x ; r) .
$$


Hence, $T x \in C$, that is, $T: C \rightarrow C$. Minimality of $K$ deduces that $C=K$. Thus, $\operatorname{diam}(K) \leq r$ which is a contradiction. Then $\operatorname{diam}(K)=0$ and hence, $K$ consists of a single point which must be a fixed point of $T$.

Remark 3.8. We note that a convex metric space $(X, d, \mathcal{W})$ need not to have the condition (D), in Theorem 3.7.

Let us illustrate Theorem 3.7 with the following example.

Example 3.9. Let $X:=[-1,1]$ and define a metric $d$ on $X$ by

$$
d(x, y)=\left\{\begin{array}{l}
0, \quad \text { if } x=y, \\
\max \{|x|,|y|\}, \quad \text { if } x \neq y
\end{array}\right.
$$

Define $\mathcal{W}: X \times X \times I \rightarrow X$ with

$$
\mathcal{W}(x, y, \lambda)=\lambda \min \{|x|,|y|\}
$$

for each $x, y \in X$ and $\lambda \in I$. We show that $\mathcal{W}$ is a convex structure on $X$. Let $x, y \in X$ and $\lambda \in I$. We may assume that $|x| \leq|y|$. Then for each $u \in X$ we have

$$
\begin{gathered}
d(u, \mathcal{W}(x, y, \lambda))=\max \{|u|, \lambda \min \{|x|,|y|\}\} \\
=\max \{|u|, \lambda|x|\} \leq \max \{|u|,|x|\} \\
=\lambda \max \{|u|,|x|\}+(1-\lambda) \max \{|u|,|x|\} \\
\leq \lambda \max \{|u|,|x|\}+(1-\lambda) \max \{|u|,|y|\} \\
=\lambda d(u, x)+(1-\lambda) d(u, y) .
\end{gathered}
$$

This implies that $(X, d, \mathcal{W})$ is a convex metric space. Now, let $E$ be a nonempty convex subset of $X$. Then $\mathcal{W}(x, y, \lambda) \in E$ for each $x, y \in E$ and $\lambda \in I$. If $\lambda=0$, then we conclude that $0 \in E$. Therefore, the convex metric space $(X, d, \mathcal{W})$ must be have the property (C). Suppose that $A:=[0,1]$. Thus, $A$ is a bounded closed and convex subset of $X$. Note that every convergent sequence in this metric space converges to $0 \in A$. Let $T: A \rightarrow A$ be a mapping defined as

$$
T x= \begin{cases}0, & \text { if } x=1 \\ x, & \text { if } x \neq 1 .\end{cases}
$$

We claim that $T$ is strongly C-nonexpansive. For this purpose it is sufficient to consider $x=1$ and $y \neq 1$. Then $d(T x, T y)=y, d(x, T y)=1$ and $d(y, T x)=y$. We now have

$$
d(T x, T y) \leq \min \{d(x, T y), d(y, T x)\} .
$$

It now follows from Theorem 3.7 that $T$ has a fixed point. It is interesting to note that the existence of fixed point for the mapping $T$ cannot be obtained from Theorem 2.12 due to Takahashi because of the mapping $T$ is not continuous.

We now raise the next problem.

Question 3.1. It is interesting to ask whether Theorem 3.4 holds whenever $T$ is noncyclic relatively C-nonexpansive. 


\section{Additional Results}

In this section, we study the structure of minimal sets of strongly noncyclic relatively $\mathrm{C}$-nonexpansive in the setting of convex metric spaces. We begin our main results of this section with the following existence theorem.

Theorem 4.1. Let $(A, B)$ be a nonempty, bounded, closed and convex pair in a convex metric space $(X, d, \mathcal{W})$ such that $X$ has the properties $(C)$ and $(D)$. Assume that $T: A \cup B \rightarrow A \cup B$ is a noncyclic mapping such that

$$
d(T x, T y) \leq \alpha[d(x, T y)+d(y, T x)]+(1-2 \alpha) \operatorname{dist}(A, B),
$$

for some $\alpha \in\left(0, \frac{1}{2}\right)$ and for all $(x, y) \in A \times B$. Then $T$ has a best proximity pair.

Proof. By using Zorn's Lemma and by the fact that $X$ has the property (C), we obtain a nonempty, closed and convex pair $\left(K_{1}, K_{2}\right)$ in $X$ which is minimal with respect to being invariant under noncyclic mapping $T$. So, we must have $K_{1}=\overline{\operatorname{cov}}\left(T\left(K_{1}\right)\right)$ and $K_{2}=\overline{\operatorname{cov}}\left(T\left(K_{2}\right)\right)$. Take $a \in K_{1}$. Then $K_{2} \subseteq \mathcal{B}\left(a ; \delta_{a}\left(K_{2}\right)\right)$. Now, if $y \in K_{2}$ then

$$
\begin{aligned}
d(T a, T y) & \leq \alpha[d(a, T y)+d(T y, a)]+(1-2 \alpha) \operatorname{dist}(A, B) \\
& \leq 2 \alpha \delta\left(K_{1}, K_{2}\right)+(1-2 \alpha) \operatorname{dist}(A, B) .
\end{aligned}
$$

Put $\rho:=2 \alpha \delta\left(K_{1}, K_{2}\right)+(1-2 \alpha) \operatorname{dist}(A, B)$. Then $T y \in \mathcal{B}(T a ; \rho)$ for each $y \in K_{2}$ and so,

$$
K_{2}=\overline{\operatorname{cov}}\left(T\left(K_{2}\right)\right) \subseteq \mathcal{B}(T a ; \rho)
$$

which deduces that

$$
\delta_{T a}\left(K_{2}\right) \leq \rho, \quad \forall a \in K_{1}
$$

Similar argument concludes that for each $b \in K_{2}$ we have $\delta_{T b}\left(K_{1}\right) \leq \rho$. Set

$$
E_{1}:=\left\{x \in K_{1}: \delta_{x}\left(K_{2}\right) \leq \rho\right\}, \quad E_{2}:=\left\{y \in K_{2}: \delta_{y}\left(K_{1}\right) \leq \rho\right\} .
$$

Note that $T\left(K_{1}\right) \subseteq E_{1}$ and $T\left(K_{2}\right) \subseteq E_{2}$. On the other hand, it is easy to verify that

$$
E_{1}=\left[\bigcap_{y \in K_{2}} \mathcal{B}(y ; \rho)\right] \cap K_{1}, \quad E_{2}=\left[\bigcap_{x \in K_{1}} \mathcal{B}(x ; \rho)\right] \cap K_{2},
$$

that is, $\left(E_{1}, E_{2}\right)$ is a closed and convex pair in $X$. Let $x \in E_{1}$. Since $\delta_{T x}\left(K_{2}\right) \leq \rho$, we have $T x \in E_{1}$, i.e., $T\left(E_{1}\right) \subseteq E_{1}$. Similarly, we have $T\left(E_{2}\right) \subseteq E_{2}$. Hence, $T$ is noncyclic on $E_{1} \cup E_{2}$. Now, by the minimality of $\left(K_{1}, K_{2}\right)$ we must have $E_{1}=K_{1}$ and $E_{2}=K_{2}$. Thus,

$$
\delta_{x}\left(K_{2}\right) \leq \rho=2 \alpha \delta\left(K_{1}, K_{2}\right)+(1-2 \alpha) \operatorname{dist}(A, B), \quad \forall x \in K_{1} .
$$

Therefore,

$$
\delta\left(K_{1}, K_{2}\right)=\operatorname{dist}(A, B) .
$$

By the fact that the convex metric space $X$ has the property (D) we conclude that both $K_{1}$ and $K_{2}$ are singleton. The conclusion then trivially follows. 
Remark 4.2. Theorem 4.1 holds once the minimal sets $K_{1}$ and $K_{2}$ have been fixed and the noncyclic mapping $T: A \cup B \rightarrow A \cup B$ satisfies the following condition.

$$
d(T x, T y) \leq r \delta\left(K_{1}, K_{2}\right)+(1-r) \operatorname{dist}(A, B),
$$

for some $r \in(0,1)$ and for all $(x, y) \in K_{1} \times K_{2}$.

The next lemma guarantees the existence of diametral pairs for strongly noncyclic relatively $\mathrm{C}$-nonexpansive mappings.

Lemma 4.3. Let $(A, B)$ be a nonempty, bounded, closed and convex pair of a convex metric space $(X, d, \mathcal{W})$ such that $X$ has the properties $(C)$ and $(D)$. Let $T: A \cup B \rightarrow A \cup B$ be a strongly noncyclic relatively $C$-nonexpansive mapping and let $\left(K_{1}, K_{2}\right) \subseteq(A, B)$ be a minimal closed and convex pair which is $T$-invariant and such that $\operatorname{dist}\left(K_{1}, K_{2}\right)=\operatorname{dist}(A, B)$. Then each $\left(x^{\star}, y^{\star}\right) \in K_{1} \times K_{2}$ with $d\left(x^{\star}, y^{\star}\right)=\operatorname{dist}(A, B)$ is a diametral pair (with respect to $\left(K_{1}, K_{2}\right)$ ), that is,

$$
\delta_{x^{\star}}\left(K_{2}\right)=\delta_{y^{\star}}\left(K_{1}\right)=\delta\left(K_{1}, K_{2}\right) .
$$

Proof. We have $K_{1}=\overline{\operatorname{cov}}\left(T\left(K_{1}\right)\right)$ and $K_{2}=\overline{\operatorname{cov}}\left(T\left(K_{2}\right)\right)$. Assume that there exists a pair $\left(x^{\star}, y^{\star}\right) \in K_{1} \times K_{2}$ with $d\left(x^{\star}, y^{\star}\right)=\operatorname{dist}(A, B)$ which is not diametral pair. Then

$$
\min \left\{\delta_{x^{\star}}\left(K_{2}\right), \delta_{y^{\star}}\left(K_{1}\right)\right\}<\delta\left(K_{1}, K_{2}\right) .
$$

Suppose that $r_{1}:=\delta_{x^{\star}}\left(K_{2}\right)<\delta\left(K_{1}, K_{2}\right)$ and $r_{2}=\delta_{y^{\star}}\left(K_{1}\right)$. Set

$$
\mathcal{C}_{r_{1}}\left(K_{2}\right):=K_{1} \bigcap\left(\cap_{x \in K_{2}} \mathcal{B}\left(T x ; r_{1}\right)\right) \quad \& \quad \mathcal{C}_{r_{2}}\left(K_{1}\right):=K_{2} \bigcap\left(\cap_{x \in K_{1}} \mathcal{B}\left(T x ; r_{2}\right)\right) .
$$

It is easy to see that $(x, y) \in \mathcal{C}_{r_{1}}\left(K_{2}\right) \times \mathcal{C}_{r_{2}}\left(K_{1}\right)$ if and only if

$$
K_{2}=\overline{\operatorname{cov}}\left(T\left(K_{2}\right)\right) \subseteq \mathcal{B}\left(x ; r_{1}\right), K_{1}=\overline{\operatorname{cov}}\left(T\left(K_{1}\right)\right) \subseteq \mathcal{B}\left(y ; r_{2}\right) .
$$

Also, $\left(x^{\star}, y^{\star}\right) \in \mathcal{C}_{r_{1}}\left(K_{2}\right) \times \mathcal{C}_{r_{2}}\left(K_{1}\right)$ and so,

$$
\operatorname{dist}\left(\mathcal{C}_{r_{1}}\left(K_{2}\right), \mathcal{C}_{r_{2}}\left(K_{1}\right)\right)=\operatorname{dist}(A, B) .
$$

Moreover, $T$ is noncyclic on $\mathcal{C}_{r_{1}}\left(K_{2}\right) \cup \mathcal{C}_{r_{2}}\left(K_{1}\right)$. Indeed, if $p \in \mathcal{C}_{r_{1}}\left(K_{2}\right)$ then $p \in K_{1}$ and for each $y \in K_{2}$ we have $d(p, T y) \leq r_{1}$. Since $T$ is strongly noncyclic relatively $\mathrm{C}$-nonexpansive,

$$
d(T p, T y) \leq \min \{d(p, T y), d(y, T p)\} \leq r_{1}, \quad \forall y \in K_{2} .
$$

Thus,

$$
K_{2}=\overline{\operatorname{cov}}\left(T\left(K_{2}\right)\right) \subseteq \mathcal{B}\left(T p ; r_{1}\right),
$$

which implies that $T p \in \mathcal{C}_{r_{1}}\left(K_{2}\right)$. Then $T\left(\mathcal{C}_{r_{1}}\left(K_{2}\right)\right) \subseteq \mathcal{C}_{r_{1}}\left(K_{2}\right)$. Similarly, $T\left(\mathcal{C}_{r_{2}}\left(K_{1}\right)\right) \subseteq \mathcal{C}_{r_{2}}\left(K_{1}\right)$. That is, $T$ is noncyclic on $\mathcal{C}_{r_{1}}\left(K_{2}\right) \cup \mathcal{C}_{r_{2}}\left(K_{1}\right)$. It now follows from the minimality of $\left(K_{1}, K_{2}\right)$ that $K_{1}=\mathcal{C}_{r_{1}}\left(K_{2}\right)$ and $K_{2}=\mathcal{C}_{r_{2}}\left(K_{1}\right)$. Thereby, $K_{2} \subseteq \bigcap_{x \in K_{1}} \mathcal{B}\left(T x ; r_{1}\right)$. Hence,

$$
\delta_{y}\left(T\left(K_{1}\right)\right) \leq r_{1}, \quad \forall y \in K_{2} .
$$

So, $\delta\left(T\left(K_{1}\right), K_{2}\right) \leq r_{1}$. By using Lemma 2.8 we obtain

$$
\delta\left(K_{1}, K_{2}\right)=\delta\left(\overline{\operatorname{cov}}\left(T\left(K_{1}\right)\right), K_{2}\right)=\delta\left(T\left(K_{1}\right), K_{2}\right) \leq r_{1},
$$

which is a contradiction. 
Definition 4.4. ([6]) Let $(A, B)$ be a nonempty pair in a metric space $(X, d)$ and $T: A \cup B \rightarrow A \cup B$ a noncyclic mapping. Then a sequence $\left(\left\{x_{n}\right\},\left\{y_{n}\right\}\right)$ in $A \times B$ is said to be an approximate best proximity pair sequence of the noncyclic mapping $T$ provided that

$$
\lim _{n \rightarrow \infty} d\left(x_{n}, T x_{n}\right)=0, \quad \lim _{n \rightarrow \infty} d\left(y_{n}, T y_{n}\right)=0 \quad \text { and } \quad \lim _{n \rightarrow \infty} d\left(x_{n}, y_{n}\right)=\operatorname{dist}(A, B) \text {. }
$$

Lemma 4.5. Let $(A, B)$ be a nonempty, bounded, closed and convex pair of a convex metric space $(X, d, \mathcal{W})$ such that $X$ has the properties $(C)$ and $(D)$. Suppose that $A_{0}$ is nonempty and $(A, B)$ is a proximal compactness pair. Let $T: A \cup B \rightarrow A \cup B$ be a strongly noncyclic relatively $C$-nonexpansive mapping. Then $T$ has an approximate best proximity pair sequence in $A \times B$.

Proof. As in the proof of Theorem 3.4, there exists a pair $\left(K_{1}, K_{2}\right) \subseteq(A, B)$ which is minimal with respect to being nonempty, closed, convex and $T$ invariant and $\operatorname{dist}\left(K_{1}, K_{2}\right)=\operatorname{dist}(A, B)$. Also, there exists $(p, q) \in K_{1} \times K_{2}$ such that $d(p, q)=\operatorname{dist}\left(K_{1}, K_{2}\right)$. For each $t \in\left(0, \frac{1}{2}\right)$ define $T_{t}: K_{1} \cup K_{2} \rightarrow K_{1} \cup K_{2}$ as follows:

$$
T_{t}(x)= \begin{cases}\mathcal{W}(T x, p, 2 t) ; & x \in K_{1}, \\ \mathcal{W}(T x, q, 2 t) ; & x \in K_{2} .\end{cases}
$$

Note that $T$ is noncyclic on $K_{1} \cup K_{2}$. Let $r:=4 t-4 t^{2}$. Since $t \in\left(0, \frac{1}{2}\right)$, we conclude $r<1$. Now for each $(x, y) \in K_{1} \times K_{2}$ we have

$$
\begin{gathered}
d\left(T_{t} x, T_{t} y\right)=d(\mathcal{W}(T x, p, 2 t), \mathcal{W}(T y, q, 2 t)) \\
\leq 2 t d(T x, \mathcal{W}(T y, q, 2 t)+(1-2 t) d(p, \mathcal{W}(T y, q, 2 t)) \\
\leq 2 t[2 t d(T x, T y)+(1-2 t) d(T x, q)]+(1-2 t)[2 t d(p, T y)+(1-2 t) d(p, q)] \\
\leq 2 t[2 t \min \{d(x, T y), d(y, T x)\}+(1-2 t) d(T x, q)] \\
+(1-2 t)[2 t d(p, T y)+(1-2 t) \operatorname{dist}(A, B)] \\
\leq 2 t\left[2 t \delta\left(K_{1}, K_{2}\right)+(1-2 t) \delta\left(K_{1}, K_{2}\right)\right]+(1-2 t)\left[2 t \delta\left(K_{1}, K_{2}\right)+(1-2 t) \operatorname{dist}(A, B)\right] \\
=\left(4 t-4 t^{2}\right) \delta\left(K_{1}, K_{2}\right)+\left(1-\left(4 t-4 t^{2}\right)\right) \operatorname{dist}(A, B) \\
=r \delta\left(K_{1}, K_{2}\right)+(1-r) \operatorname{dist}(A, B) .
\end{gathered}
$$

It now follows from Remark 4.2 that $T_{t}$ has a best proximity pair for each $t \in$ $\left(0, \frac{1}{2}\right)$, that is, there exists $\left(x_{t}, y_{t}\right) \in K_{1} \times K_{2}$ such that

$$
x_{t}=T_{t}\left(x_{t}\right), \quad y_{t}=T_{t}\left(y_{t}\right) \quad \text { and } \quad d\left(x_{t}, y_{t}\right)=\operatorname{dist}(A, B), \forall t \in\left(0, \frac{1}{2}\right) .
$$

We now have

$$
\begin{gathered}
d\left(x_{t}, T x_{t}\right)=d\left(T_{t}\left(x_{t}\right), T x_{t}\right) \\
=d\left(\mathcal{W}\left(T x_{t}, p, 2 t\right), T x_{t}\right) \leq(1-2 t) d\left(p, T x_{t}\right) \leq(1-2 t) \operatorname{diam}(A) .
\end{gathered}
$$

This implies that $d\left(x_{t}, T x_{t}\right) \rightarrow 0$ if $t \rightarrow \frac{1}{2}^{-}$. By a similar way, we can see that $d\left(y_{t}, T y_{t}\right) \rightarrow 0$ whenever $t \rightarrow \frac{1}{2}^{-}$and this completes the proof.

Here, we state the main result of this section. 
Theorem 4.6. Let $(A, B)$ be a nonempty, bounded, closed and convex pair of a convex metric space $(X, d, \mathcal{W})$ such that $X$ has the properties $(C)$ and $(D), A_{0}$ is nonempty and $(A, B)$ is a proximal compactness pair. Let $T: A \cup B \rightarrow A \cup B$ be a strongly noncyclic relatively $C$-nonexpansive mapping. Suppose that $\left(K_{1}, K_{2}\right) \subseteq$ $(A, B)$ is a minimal, closed and convex pair which is $T$ invariant and such that $\operatorname{dist}\left(K_{1}, K_{2}\right)=\operatorname{dist}(A, B)$ and let $\left(\left\{x_{n}\right\},\left\{y_{n}\right\}\right)$ be an approximate best proximity pair sequence in $A \times B$. Then for each $(p, q) \in K_{1} \times K_{2}$ with $d(p, q)=\operatorname{dist}(A, B)$ we have

$$
\limsup _{n \rightarrow \infty} d\left(T x_{n}, q\right)=\limsup _{n \rightarrow \infty} d\left(p, T y_{n}\right)=\delta\left(K_{1}, K_{2}\right) .
$$

Proof. Lemma 4.5 guarantees that the noncyclic mapping $T$ has an approximate best proximity pair sequence in $A \times B$. By the fact that $(A, B)$ is proximal compactness, there exists a subsequence $\left(\left\{x_{n_{k}}\right\},\left\{y_{n_{k}}\right\}\right)$ of the sequence $\left(\left\{x_{n}\right\},\left\{y_{n}\right\}\right)$ such that $x_{n_{k}} \rightarrow x^{\star}$ and $y_{n_{k}} \rightarrow y^{\star}$ for some $\left(x^{\star}, y^{\star}\right) \in K_{1} \times K_{2}$. So,

$$
d\left(x^{\star}, y^{\star}\right)=\lim _{k \rightarrow \infty} d\left(x_{n_{k}}, y_{n_{k}}\right)=\operatorname{dist}(A, B) .
$$

It follows from Lemma 4.3 that $\left(x^{\star}, y^{\star}\right)$ is a diametral pair. Let $(p, q) \in K_{1} \times K_{2}$ be such that $d(p, q)=\operatorname{dist}(A, B)$. Put,

$$
r_{1}:=\limsup _{n \rightarrow \infty} d\left(T x_{n}, q\right), \quad r_{2}:=\limsup _{n \rightarrow \infty} d\left(p, T y_{n}\right) .
$$

We assert that

$$
r_{1}=r_{2}=\delta\left(K_{1}, K_{2}\right)
$$

Suppose that $r_{2}<\delta\left(K_{1}, K_{2}\right)$ and set

$$
\begin{aligned}
& L_{1}:=\left\{x \in K_{1}: \limsup _{n \rightarrow \infty} d\left(x, T y_{n}\right) \leq r_{1}\right\}, \\
& L_{2}:=\left\{y \in K_{2}: \limsup _{n \rightarrow \infty} d\left(T x_{n}, y\right) \leq r_{2}\right\} .
\end{aligned}
$$

Then $(p, q) \in L_{1} \times L_{2}$ and it is easy to see that $\left(L_{1}, L_{2}\right)$ is a closed pair in $X$. Moreover, if $x_{1}, x_{2} \in L_{1}$ and $\alpha \in(0,1)$, then

$$
\limsup _{n \rightarrow \infty} d\left(\mathcal{W}\left(x_{1}, x_{2}, \alpha\right), T y_{n}\right) \leq \limsup _{n \rightarrow \infty}\left[\alpha d\left(x_{1}, T y_{n}\right)+(1-\alpha) d\left(x_{2}, T y_{n}\right)\right] \leq r_{1}
$$

Thus, $\mathcal{W}\left(x_{1}, x_{2}, \alpha\right) \in L_{1}$, that is, $L_{1}$ is convex. Similarly, we can see that $L_{2}$ is a convex set. Besides, if $x \in L_{1}$, then

$$
\begin{gathered}
\limsup _{n \rightarrow \infty} d\left(T x, T y_{n}\right) \leq \limsup _{n \rightarrow \infty} \min \left\{d\left(x, T y_{n}\right), d\left(y_{n}, T x\right)\right\} \\
\leq \limsup _{n \rightarrow \infty} d\left(x, T y_{n}\right) \leq r_{1},
\end{gathered}
$$

which concludes that $T x \in L_{1}$, that is, $T\left(L_{1}\right) \subseteq L_{1}$. Similarly, we can see that $T\left(L_{2}\right) \subseteq L_{2}$. Hence, $T$ is noncyclic on $L_{1} \cup L_{2}$. Minimality of $\left(K_{1}, K_{2}\right)$ deduces that $\left(K_{1}, K_{2}\right)=\left(L_{1}, L_{2}\right)$. Then for each $y \in K_{2}$ we have

$$
\begin{gathered}
d\left(x^{\star}, T y\right)=\lim _{k \rightarrow \infty} d\left(x_{n_{k}}, T y\right) \\
\leq \limsup _{n \rightarrow \infty} d\left(x_{n}, T y\right) \leq \limsup _{n \rightarrow \infty}\left[d\left(x_{n}, T x_{n}\right)+d\left(T x_{n}, T y\right)\right] \\
=\limsup _{n \rightarrow \infty} d\left(T x_{n}, T y\right) \leq \limsup _{n \rightarrow \infty} \min \left\{d\left(x_{n}, T y\right), d\left(y, T x_{n}\right)\right\}
\end{gathered}
$$




$$
\leq \limsup _{n \rightarrow \infty} d\left(y, T x_{n}\right) \leq r_{2} .
$$

Hence, $\delta_{x^{\star}}\left(T\left(K_{2}\right)\right) \leq r_{2}$. Now, by using Lemma 2.9 we conclude that

$$
\delta_{x^{\star}}\left(K_{2}\right)=\delta_{x^{\star}}\left(\overline{\operatorname{cov}}\left(T\left(K_{2}\right)\right)\right)=\delta_{x^{\star}}\left(T\left(K_{2}\right)\right) \leq r_{2}<\delta\left(K_{1}, K_{2}\right),
$$

which is a contradiction by the fact that $\left(x^{\star}, y^{\star}\right)$ is a diametral pair. By the similar way, we can see that if $r_{1}<\delta\left(K_{1}, K_{2}\right)$, then we get a contradiction.

The following corollary is immediate from the proof of Theorem 4.6.

Corollary 4.7. Under the conditions of Theorem 4.6 if, in addition, the sequence $\left\{x_{n}\right\}$ is converges to $x^{\star} \in A$, then $T$ has a best proximity pair.

Proof. By Theorem 4.6 we have

$$
\begin{gathered}
\delta\left(K_{1}, K_{2}\right)=\lim _{n \rightarrow \infty} d\left(T x_{n}, y^{\star}\right) \leq \lim _{n \rightarrow \infty}\left[d\left(T x_{n}, x_{n}\right)+d\left(x_{n}, y^{\star}\right)\right] \\
=d\left(x^{\star}, y^{\star}\right)=\operatorname{dist}\left(K_{1}, K_{2}\right) .
\end{gathered}
$$

Now, by the fact that the convex metric space $X$ has the property (D), we conclude that $K_{1}$ and $K_{2}$ are singleton and the result follows.

Corollary 4.8. Let $(A, B)$ be a nonempty, weakly compact and convex pair of a strictly convex Banach space $X$. Let $T: A \cup B \rightarrow A \cup B$ be a strongly noncyclic relatively $C$-nonexpansive mapping. Suppose that $\left(K_{1}, K_{2}\right) \subseteq(A, B)$ is a minimal, closed and convex pair which is $T$ invariant and such that $\operatorname{dist}\left(K_{1}, K_{2}\right)=$ $\operatorname{dist}(A, B)$ and let $\left(\left\{x_{n}\right\},\left\{y_{n}\right\}\right)$ be an approximate best proximity pair sequence in $A \times B$. Then for each $(p, q) \in K_{1} \times K_{2}$ with $d(p, q)=\operatorname{dist}(A, B)$ we have

$$
\limsup _{n \rightarrow \infty} d\left(T x_{n}, q\right)=\limsup _{n \rightarrow \infty} d\left(p, T y_{n}\right)=\delta\left(K_{1}, K_{2}\right) .
$$

The next corollary is similar to the classical Goebel-Karlovitz Lemma which is a key lemma in fixed point theory.

Corollary 4.9. Let $A$ be a nonempty, bounded, closed and convex subset of a convex metric space $(X, d, \mathcal{W})$ and let $T: A \rightarrow A$ be a strongly $C$-nonexpansive mapping. Assume that $K$ is a subset of $A$ which is minimal with respect to being nonempty, closed, convex and T-invariant, and suppose $\left\{x_{n}\right\}$ is a sequence in $K$ such that

$$
\lim _{n \rightarrow \infty} d\left(x_{n}, T x_{n}\right)=0 .
$$

Then, for each $x \in K, \lim _{n \rightarrow \infty} d\left(x, T x_{n}\right)=\operatorname{diam}(K)$.

Acknowledgement. The author would like to thank the reviewer for his/her suggestions. 


\section{REFERENCES}

1. A. Abkar and M. Gabeleh, Global optimal solutions of noncyclic mappings in metric spaces, J. Optim. Theory Appl. 153 (2012), 298-305.

2. A. Abkar and M. Gabeleh, Proximal quasi-normal structure and a best proximity point theorem, J. Nonlin. Convex Anal. 14 (2013), 653-659.

3. B. Ali and G.C. Ugwunnadi, A general iterative algoritm for nonexpansive mappings in Banach spaces, Ann. Funct. Anal. 2 (2011), 10-21.

4. A. Betiuk-Pilarska and A. Wisnicki, On the Suzuki nonexpansive-type mappings, Ann. Funct. Anal. 4 (2013), 72-86.

5. A.A. Eldred, W.A. Kirk and P. Veeramani, Proximal normal structure and relatively nonexpansive mappings, Studia Math. 171 (2005), 283-293.

6. R. Espinola and M. Gabeleh, On the structure of minimal sets of relatively nonexpnsive mappings, Numer. Funct. Anal. Optim. 34 (2013), 845-860.

7. M.S. Brodskii and D.P. Milman, On the center of a convex set (Russian), Dokl. Akad. Nauk. USSR 59 (1948), 837-840.

8. S.K. Chatterjea, Fixed Point Theorems, C.R. Acad. Bulgare Sci. 25 (1972), 727-730.

9. M. Gabeleh, Minimal sets of noncyclic relatively nonexpansive mappings in convex metric spaces, Fixed Point Theory (to appear).

10. K. Goebel, On the structure of minimal invariant sets for nonexpansive mappings, Ann. Univ. Mariae Curie-Skłodowska Sect. A 29 (1975), 73-77.

11. L. Karlovitz, Existence of fixed point for nonexpansive mappings in spaces without normal structure, Pacific J. Math. 66 (1976), 153-156.

12. M.A. Khamsi and W.A. Kirk, An Introduction to Metric Spaces and Fixed Point Theory, Pure and Applied Mathematics, Wiley-Interscience, New York, USA, 2001.

13. W.A. Kirk, A fixed point theorem for mappings which do not increase distances, Amer. Math. Monthly 72 (1965), 1004-1006.

14. J. Matkowski, Remarks on Lipschitzian mappings and some fixed point theorems, Banach J. Math. Anal. 1 (2007), 237-244.

15. T. Shimizu and W. Takahashi, Fixed points of multivalued mappings in certian convex metric spaces, Topol. Methods Nonlinear Anal. 8 (1996), 197-203.

16. Y. Su, M. Shang and D. Wang, Strong convergence of monotone CQ algorithm for relatively nonexpansive mappings, Banach J. Math. Anal. 2 (2008), 1-10.

17. W. Takahashi $A$ convexity in metric space and nonexpansive mappings, Kodai Math. Sem. Rep. 22 (1970), 142-149.

Department of Mathematics, Ayatollah Boroujerdi University, Boroujerd, IRAN.

E-mail address: gab.moo@gmail.com, Gabeleh@abru.ac.ir 